\title{
PRELIMINARY RESULTS FROM A SCALED FINAL FOCUS EXPERIMENT FOR HEAVY ION INERTIAL FUSION*
}

\author{
$\underline{\text { S. MacLaren }}{ }^{\#}$, A. Faltens, E. Henestroza, G. Ritchie, P. Seidl, Lawrence Berkeley National \\ Laboratory, Berkeley, CA 94720
}

\begin{abstract}
Vacuum ballistic focusing may be a straightforward method to obtain the heavy ion beam spot size necessary to drive an inertial confinement fusion target. Proper scaling of particle energy, mass, beam current, beam emittance, and magnetic field replicates the dynamics of a full driver beam in a small laboratory experiment. By expanding the beam and then focusing to a very small spot, the effects of aberrations and space charge on this method of final focus can be studied. To date, $200 \mu \mathrm{A}$ of $120 \mathrm{keV} \mathrm{K} \mathrm{K}^{+}$has been focused to test the matching and focusing elements. A recently designed high brightness contact ionization source is being tested for upcoming focusing measurements with an $87 \mu \mathrm{A}$ Cs beam that will duplicate the dynamics of a proposed driver design at onetenth scale. Transverse phase space and beam current density at various stages of the focus will be presented. Follow-on measurements studying electron neutralization of space charge and its effect on the focus will be explored.
\end{abstract}

\section{BACKGROUND AND MOTIVATION}

There are several important challenges associated with the final focus system design in a heavy ion fusion (HIF) driver. A combination of target design and the performance of the final focusing section determine the emittance budget for the accelerator. Additionally, for many accelerator designs there is a lower limit of approximately $10 \mathrm{GeV}$ for the ion kinetic energy in an unneutralized vacuum focus scenario [1]. This statement is troublesome when considering the economic expense of achieving high ion kinetic energy in an induction accelerator. Combined with the limitations of current fusion target requirements, this indicates a need for a detailed experimental study of final focus performance.

Proper scaling of the terms in the beam envelope equation that relate particle energy and mass, beam current, emittance, and focusing field replicates the dynamics of a full driver beam in a small laboratory beam. The experiment can be scaled to reproduce the beam conditions in a full-scale driver, including aberrations from the focusing elements and the longitudinal momentum spread. In addition to partial neutralization from the reactor chamber gas and secondary electrons from beam target interactions, current reactor scenarios include deliberate methods for reducing the perveance of the beams incident on the HIF target. Controlled neutralization of the experimental beam may be used to explore proposed methods to bring higher perveance beams to a small focal spot. These additional effects, which are very difficult to model accurately in simulations, must be studied closely in order to advance a valid design for the final focus subsystem.

\section{DESCRIPTION OF THE EXPERIMENT}

A Scaled Final Focus Experiment has been built at Lawrence Berkeley National Laboratory to investigate the ballistic focus of a heavy ion beam using a series of pulsed magnetic quadrupoles. The final focus system designed by H. Wollnik for the HIBALL II study [2] used $10 \mathrm{GeV} \mathrm{Bi}^{+}$and has been experimentally reproduced with $160 \mathrm{keV} \mathrm{Cs}^{+}$at a one-tenth scale. Accordingly, the beam current is scaled from $1.25 \mathrm{kA}$ to $87 \mu \mathrm{A}$. The beam is apertured and matched to the entrance of the final focus section in a 1.5-meter lattice of ten electrostatic quadrupoles. The magnetic focusing section is 8.2 meters long, including initial and final drifts. The middle drift between the third and fourth magnetic quadrupoles has been shortened with respect to the HIBALL II design as the bending magnets that allow for the neutron dump are not included.

A schematic of the experiment along with the beam envelope is shown in Figure 1. The upper part of the figure contains a to-scale drawing of the vacuum system with electrostatic quadrupoles in pink and magnetic quadrupoles in green. The lower part is a schematic of the horizontal (red) and vertical (blue) beam envelope with the transverse scale exaggerated. The dashed lines represent the locations of the diagnostic probes, and the solid black line represents the aperture location.

The source for the $\mathrm{Cs}^{+}$ion beam is a sintered iridium emitter that can be replenished with $\mathrm{Cs}$ atoms using an aqueous $\mathrm{Cs}_{2} \mathrm{CO}_{3}$ solution. A method for replenishing the emitter under vacuum using a Cs vapor feed is currently being investigated. The emitter sits in a diode with a Pierce cathode geometry that extracts $8 \mathrm{~mA} / \mathrm{cm}^{2} \mathrm{Cs}^{+}$when pulsed at $160 \mathrm{kV}$. The design and performance of the source are discussed in detail elsewhere in these proceedings [3].

\footnotetext{
*Work supported by the Director, Office of Energy Research, Office of Fusion Energy, U.S. DOE, under Contract No. DE-AC03-76SF00098.

\# email: samaclaren@lbl.gov
} 


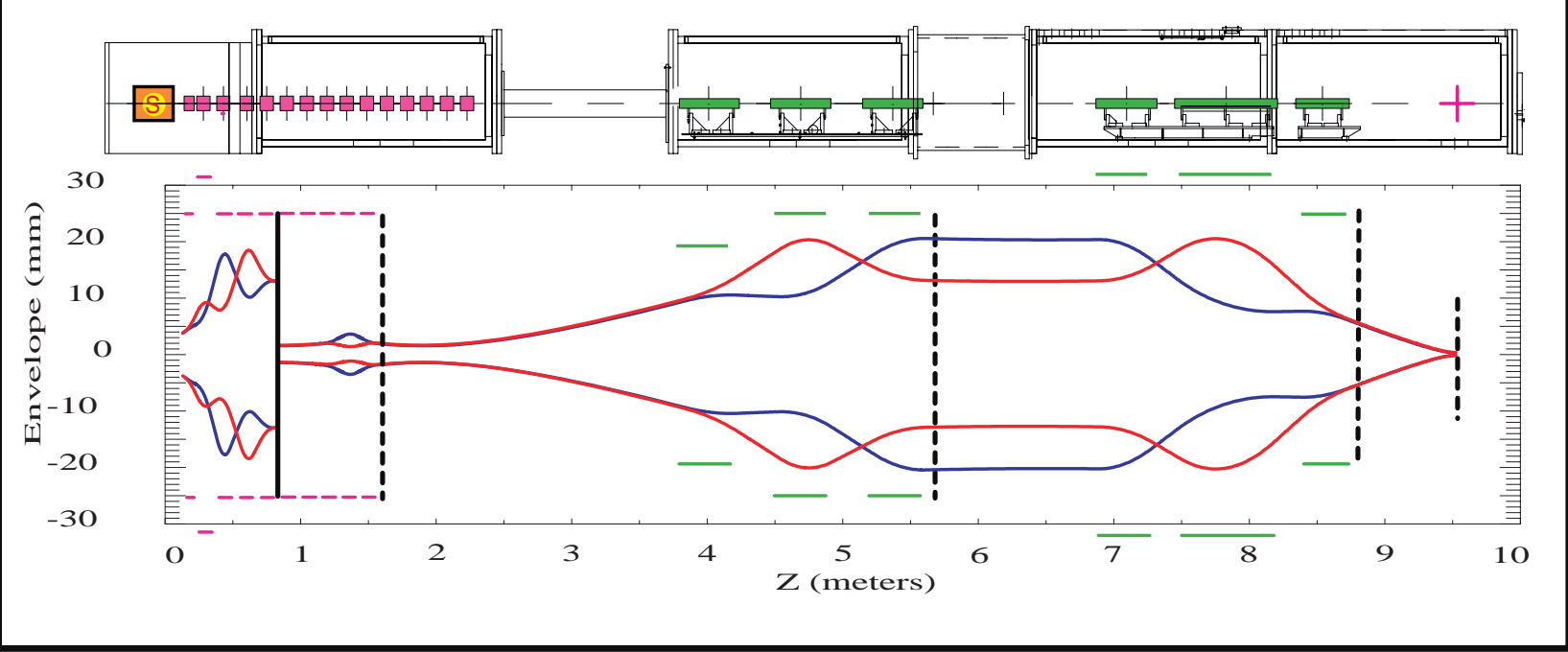

Figure 1: Experimental layout and beam envelopes.

Upon exit from the diode, $6.3 \mathrm{~mA}$ of $\mathrm{Cs}^{+}$is transported through the first set of five electrostatic quadrupoles to an aperture plate. A 3-mm diameter aperture passes $87 \mu \mathrm{A}$ of beam into the second set of five quadrupoles, which allow the beam to expand and then give it an overall convergence of a few milliradians in both transverse directions for the drift into the magnetic section. The final focus lattice consists of six pulsed magnetic quadrupoles, each with two layers of copper windings in a $\cos 2 \theta$ distribution on cylindrical shells. Several of the magnets are pictured in Figure 2, and their properties are summarized in Table 1 . The beam leaves the sixth magnetic quadrupole with a converging $(-9 \mathrm{mr})$ envelope angle and reaches its focal point approximately $80 \mathrm{~cm}$ from the last magnet.

\begin{tabular}{|l|l|l|}
\hline Magnet & Effective length (cm) & Pole Tip Field (gauss) \\
\hline QM1 & $\mathbf{4 0 . 0}$ & $\mathbf{3 1 0}$ \\
\hline QM2 & $\mathbf{4 0 . 0}$ & $\mathbf{5 9 6}$ \\
\hline QM3 & $\mathbf{4 0 . 0}$ & $\mathbf{3 4 6}$ \\
\hline QM4 & $\mathbf{4 0 . 0}$ & $\mathbf{4 0 2}$ \\
\hline QM5 & $\mathbf{7 1 . 7}$ & $\mathbf{4 4 0}$ \\
\hline QM6 & $\mathbf{3 5 . 1}$ & $\mathbf{6 6 5}$ \\
\hline
\end{tabular}

Table 1: Magnetic quadrupole properties

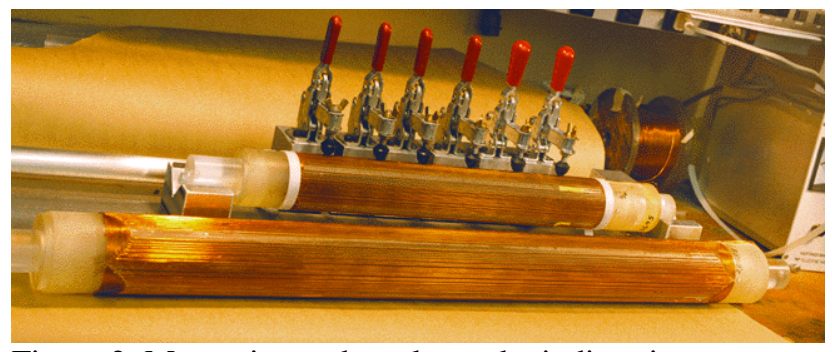

Figure 2: Magnetic quadrupoles and winding rig

\section{RECENT DATA}

Thus far, a 0.86-mm radius focal spot has been achieved with a $200-\mu \mathrm{A}$ beam of $120 \mathrm{keV} \mathrm{K} \mathrm{K}^{+}$ions from an aluminosilicate source that had been used in experiments several years ago. Figure 3 shows the beam profile measured with a single $50 \mu$ slit at the focus as a function of the duration of the pulse. At each time slice, the data are well represented by two Gaussian curves, one broad with low amplitude, and another narrower with larger amplitude. The widths of these Gaussians are plotted in the lower half of the figure.

The size of the focus compares very well with the calculated value $(0.88 \mathrm{~mm})$ of for the minimum of the beam envelope. This value was obtained by integrating the envelope equation from the exit of the last magnet to the focus using the measured beam emittance and convergence angle. For the calculation, the beam current corresponding to the core Gaussian $(142 \mu \mathrm{A})$ was used.

As the full $10-\mathrm{mA}$ beam from the $\mathrm{K}^{+}$source was highly non-uniform, it was not expected that the apertured beam would be perfectly uniform. In fact, current density and phase space measurements made of the apertured beam 80 $\mathrm{cm}$ from the aperture demonstrate this non-uniformity. The lower amplitude Gaussian represents a beam halo most likely resulting from the non uniformity of the current distribution emerging from the aperture. The high degree of uniformity from the newly tested sintered 
iridium source will remove this possible aberration from the focal spot measurement [3].

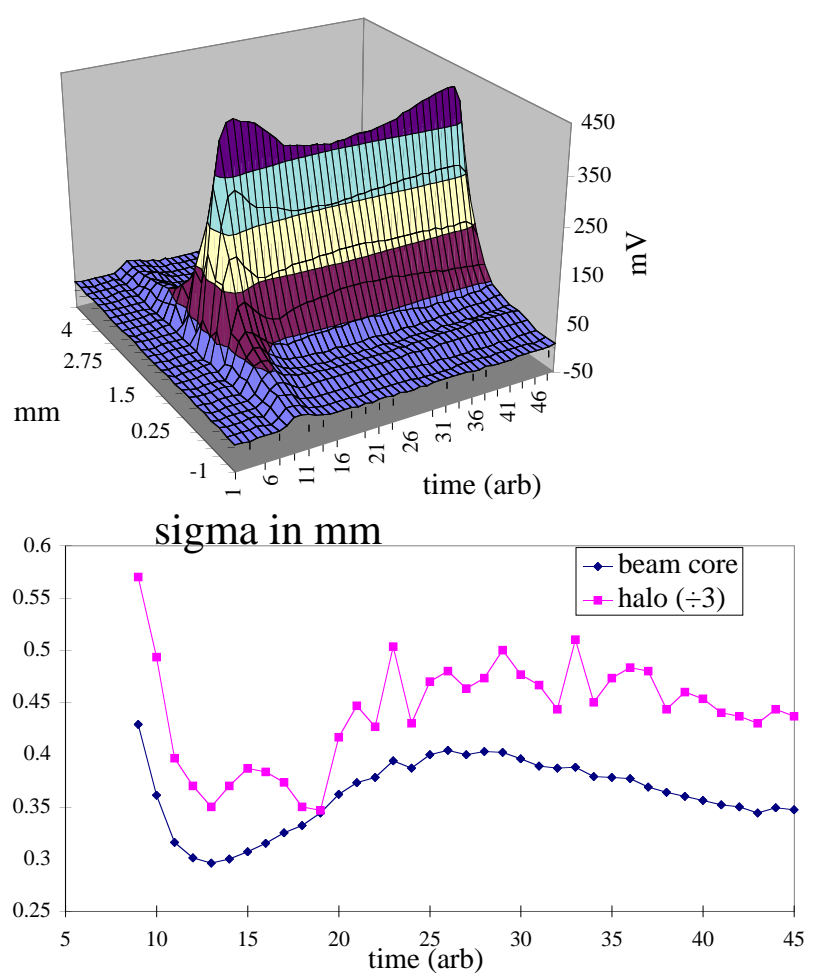

Figure 3: Beam profile dataat focus

The time dependence of the beam pulse shows a monotonic decreaseof the spot radius from the to the end. The measurement was made with a steel diagnostic slit that intercepted the beam just upstream of a Faraday cup. This time dependenteffect is most likely due to secondary electrons producedby the unbiased slit paddle neutralizing the incident beam. The decrease to $0.74-\mathrm{mm}$ radius is consistent with a $42 \%$ neutralization of the space charge. A diagnostic probe that includes secondary-electron suppression and is capable of three-axis motion is currently being manufactured.

\section{FUTURE PLANS}

Once the apertured $\mathrm{Cs}^{+}$beam is brought to a focus, the next step in the evaluation of the HIBALL-IIstudy will be to match the emittance of the experiment to the corresponding scaled value of $3 \pi \mathrm{mm} \mathrm{mr}$. This may be done with the use of an emittance-spoiling grid at the diode exit, or possibly with a poor match in the electrostatic section. A comparison of the measured spot size with the study's results can then be made.

An effort to simulate the experiment with a 3-D PIC code is currently underway. The quadrupole end fields of the magnets will be included in the PIC simulations in order to make comparisons with the measured phase space and the focal spot profiles.

Beyond this, there are two more studies that will be carriedout in the near future. First, there is the question of chromatic aberrations caused by the magnets due to deviations from the design longitudinal momentum. This effectcan be studied by deliberately detuning the magnetic quadrupole fields to alter the forces on the particles, simulating a deviation in their longitudinal momentum from the referencevalue. Measurements of the resulting effect on the focal spot could then be compared directly with simulation.

Second, the possibility of reducing the focal spot size by partially neutralizing the beam with electrons can be examined. Due to the brightness of the beam, a larger aperture may be used in the electrostatic section to allow approximately $400 \mu \mathrm{A}$ of beam into the final focus lattice. Increasing the beam perveance by a factor of four while only doubling the emittance moves the focus into a regime that is highly space charge dominated with a focal spot radius of several millimeters for a completely unneutralizedbeam. A high transparency grid inserted in the beam path immediately following the last magnet could produceenough secondaryelectrons to have a considerable and easily measurable effect on the beam spot size. This experimental measurement could then be compared with a separate measurement of the fractional current neutralization as well as the PIC simulation results.

\section{ACKNOWLEDGMENTS}

The authors gratefully acknowledge the outstanding support of the LBNL technical staff including Mr. R. Beggs, Mr. R. Hipple, Mr. C. Rogers, and Mr. W. Strelo.

\section{REFERENCES}

[1] R.O. Bangerter, The US Heavy Ion Fusion Program, Fus. Eng. Des., 32-33 (1996) 27-32.

[2] HIBALL-II An Improved Conceptual Heavy Ion Beam Driven Fusion Reactor Study", KfK 3840, KernforschungszentrumKarlsruhe (July 1985), pp. 57-71. [3] S. MacLaren et. al., "A High Current Density Contact Ionization Source For Heavy-Ion Fusion", these proceedings. 